\title{
Free and Bound Pro-Verbs: A Unified Treatment of Anaphora
}

\author{
Simon Charlow \\ Brown University, New York University
}

\section{Introduction}

This paper argues that certain phenomena related to verb ellipsis (VE) in English suggest a degree of interpretive flexibility for ellipsis sites close to that exhibited by pronominals. Specifically, it is claimed that (a) like pronouns, ellipsis sites may be bound or free, and (b) one set of grammatical mechanisms affords analyses of both free and bound pronominals and free and bound pro-verbs.

\section{Canonical Arguments for Pronominal Binding}

\subsection{Quantificational and Multiple Antecedents}

Pronouns may be interpreted non-referentially: ${ }^{1}$

(1) No $\operatorname{man}_{i}$ loves his $s_{i}$ mother.

(2) $\mathrm{John}_{i}$ loves and Bill $j$ hates his $i, j$ mother.

Example (1)'s pronominal's antecedent is a negative existential quantified phrase. There's nothing here for the pronoun to refer to, and so the quantified subject is instead taken to bind his in a manner akin to variable binding in predicate logic.

Example (2) represents a case of so-called "across-the-board" (ATB) binding. On the indicated reading, (2) means that John loves John's mother, and Bill hates Bill's mother. The pronominal, then, is simultaneously "co-referential" with two antecedents - and hence actually referential to nothing. Argument-doubling binding, in particular that characteristic of Jacobson (1999)'s variable-free logic for anaphora, is well-suited to such cases, as shown explicitly in Jacobson (1996).

\subsection{Focused Antecedents Plus only}

Sentence (3) may mean (a) Sue is the only individual $x$ who thinks Sue is smart or (b) Sue is the only individual $x$ who thinks $x$ is smart. I'll call these readings "free-focus" and "bound-focus", respectively:

Thanks to Polly Jacobson, Chris Barker, Nate Charlow, Emma Cunningham, Paul Elbourne, Kai von Fintel, Ben Russell, and Anna Szabolcsi, who provided helpful discussion and comments. This work was supported by NSF Grant BNS-0646081; PI Pauline Jacobson.

${ }^{1}$ Unless otherwise noted, indices are used only to highlight readings and possess no syntactic or semantic content in the model language. 
(3) Only [s $\mathrm{SUE}_{i}$ thinks she ${ }_{i}$ is smart]

If only ${ }^{2}$ takes a proposition $p$ and a focus set $\varphi$ (calculated for a constituent $\alpha$ by replacing the denotation of the focus-marked element in $\alpha$ with salient alternatives and collecting the resulting meanings) and returns a presupposition equivalent to $p$ and an assertion equivalent to the conjoined negations of the proper alternatives to $p$ in $\varphi$, the free-focus reading requires a focus set containing propositions of the form that $x$ thinks Sue is smart, for all $x \in D_{e}^{c}$-a contextually relativized set of individuals. The bound-focus reading, by contrast, requires an "unmixed" focus set-i.e. containing propositions of the form that $x$ thinks $x$ is smart, for all $x \in D_{e}^{c}$. If (a) semantically identifying a pronoun with some focused antecedent $A_{F}$ via free or "accidental" co-reference does not cause the pronoun to inherit the set of alternatives associated with $A_{F}$ and (b) the grammar affords an argument-doubling binding mechanism which allows thinks she is smart to characterize the set of all individuals $x$ who think $x$ is smart, the ambiguity follows naturally.

\subsection{Strict and Sloppy Identity in VPE constructions}

(4) a. Chris loves his $_{i}$ mother.

b. NATE $_{j}$ does $\left[\varnothing\right.$ love his $_{i / j / * k}$ mother] too. ${ }^{3}$

The salient issue raised by (4) is why his in (4b) may refer a non-Chris individual only if that individual is Nate. One way to approach the problem is alternative semantics. Rooth (1992)'s focus constraint on ellipsis (FCE) is such that (informally) if some part of a sentence $A$ serves as the antecedent for the elision of some part of a sentence $B, \llbracket A \rrbracket$ must be a member of $B$ 's focus set, $B_{\varphi}$.

On the strict reading of (4b) (such that Nate loves Chris's mother), $\left(4 \mathrm{~b}_{\varphi}\right)$ contains propositions of the form that $x$ loves Chris's mother, for all $x \in D_{e}^{c}$. That Chris loves Chris's mother - the denotation of (4a), roughly - is in this set, and so the FCE is satisfied. The FCE is, however, not satisfied on the starred reading (such that Nate loves, say, Ted's mother).

On the sloppy reading, the FCE is satisfied if we assume what's elided is a VP-type meaning characterizing the set of individuals $x$ such that $x$ loves $x$ 's mother. Combining this value with the alternatives to Nate yields a set containing propositions of the form that $x$ loves $x$ 's mother for all $x \in D_{e}^{c}$, of which $\llbracket 4 \mathrm{a} \rrbracket$ is a member. Argument-doubling operations like the Derived VP rule of e.g. Sag (1976) and Partee (1975) or Jacobson (1999)'s $\mathbf{z}$ make the relevant VP-type meaning available by merging the object and subject slots in the VP's functional denotation.

Setting aside the FCE, we note further that with respect to (certain) identitybased constraints on ellipsis, an argument-reduced VP is again precisely the sort of object which allows for a sloppy reading of (4b). ${ }^{4}$

\footnotetext{
${ }^{2}$ Here and throughout, boldface identifies both model-theoretic objects and CCG combinators.

${ }^{3}$ Subscripted $\varnothing$ denotes an elided constituent, and all-caps denotes focus-marking.

${ }^{4}$ Indeed, when coupled with a general mechanism for either generating ellipsis material based on antecedent material-staying agnostic for the moment as to whether this material is semantic
} 

a. Chris [vp $\lambda_{2}\left[t_{2}\right.$ loves his 2 mother] $]$
b. Nate does [ $\mathrm{vP}_{\varnothing} \lambda_{1}\left[t_{1}\right.$ loves his ${ }_{1}$ mother $\left.]\right]$ too

Here $\lambda$-operators render both antecedent and elided VPs constant over assigment functions and thus interpretively identical. Any constraint on ellipsis that enforces the interpretive identity of antecedent and ellipsis sites is accordingly satisfied.

\section{Adapting These Arguments to VE}

Though, as noted in Schwarz (2000), there don't seem to exist quantificational antecedents for ellipsis the way there exist quantificational pronominal antecedents, three of the above four arguments for pronominal binding have VE analogs.

\subsection{ATB Ellipsis-VPE and ACD}

$$
\text { John } \operatorname{ran}_{i} \text { and Mary jumped }{ }_{j} \text { after Jim did } \varnothing_{i, j}
$$$$
\text { John had to print }{ }_{i} \text { and Mary had to file }{ }_{j} \text { all the documents Sam neglected to } \varnothing_{i, j}
$$

Like example (1), the indicated readings of examples (6) and (7)—which, though somewhat marginal (especially 6), are generally tolerated by informants - cannot be obtained by referentially interpreting the elided constituent. For example, positing something like print and file in the ellipsis site of (7) yields a reading such that John is printing and Mary is filing the same set of documents-those documents Sam neglected to print and file. Intuitively, this isn't correct. What we're after instead is a reading such that John had to print the documents Sam didn't print, and Mary had to file the documents Sam didn't file. ${ }^{5}$ The framework developed below predicts the syntactic and semantic viability of these sorts of ATB ellipsis constructions and implicates binding mechanisms in their analysis.

\subsection{Bound-Focus Readings and Kratzer (1991)}

(8) I only went to TANGLEWOOD after you did.

(9) I only drove the PINK Edsel after you did.

Sentences (8) and (9), due to Kratzer (1991), seem to mean that for all $x \in D_{e}^{c}$, Tanglewood is the only $x$ s.t. I went to $x$ after you went to $x$, and for all $P \in D_{\langle e, t\rangle}^{c}$, pink is the only $P$ s.t. I drove the $P$ Edsel after you drove the $P$ Edsel.

or syntactic in nature - or for comparing to-be-elided material with antecedent material, the FCE seems to do roughly the same work of restricting possible readings as do grammatical identity-based constraints on ellipsis (we see this explicitly in the case of Schwarz (2000)), though formalizing this is beyond the scope of the present paper. Since CCG lacks levels of representation the FCE is better suited to the framework ultimately proposed herein.

${ }^{5}$ Something like print and file, respectively —inferred à la Webber (1978)—might be an acceptable representation of the elided material. I won't have more to say about this analysis. 
Imagine only as an operator which raises to take scope over its host clause. Given its semantics, the focus sets associated with the S-nodes of (8) and (9) are, it follows, populated with unmixed alternatives-i.e. for (9) propositions of the form that I drove the $P$ Edsel after you drove the $P$ Edsel, for all $P \in D_{\langle e, t\rangle}^{c}$-exactly as in the bound-focus derivation of only $S U E_{i}$ thinks she $e_{i}$ is smart. Only negates the disjunction of these alternatives, giving (8) and (9) their anti-copycat force. ${ }^{6}$

As Kratzer notes, (8) isn't such an intractable case. TANGLEWOOD might raise out at LF, binding co-indexed $e$-type traces in the antecedent and elided VPs and thereby guaranteeing an unmixed focus set. But it's harder to see how quantifier raising (QR) helps with (9); pink isn't generally treated as a movable object. Likewise in the spirit of constraining movement, Kratzer does not allow the full VP to scope over its host clause in order to bind its trace and a VP-type variable at the ellipsis site - the solution advocated in Schwarz (2000). Binding, recall, guarantees unmixed focus sets, as we observed in Section 2.2.

Kratzer instead adopts a new set of indices and assignment functions in order to generate the appropriate focus sets. I won't go into her solution other than to note that it represents what I call an "ellipsis-only" account. In such accounts (e.g. Sag (1976), Williams (1977)) ellipsis constructions are derived solely via the copying or deletion (or phonological nullification) of syntactic material under some sort of identity constraint (Kratzer, for instance, assumes a copy operation). On such accounts, crucially, the elided meaning is in general made fully explicit at LF. This is in decided contrast to pro-form accounts (e.g. Hardt (1993), Jacobson (1992a) Schwarz (2000), Szabolcsi (1992)) in which the elided constituent is not explicitly instantiated at any level of syntactic representation.

\subsubsection{Bound-Focus Readings and ACD}

Note moreover that bound-focus readings of ACD constructions seem in certain instances to be viable (we return to these below):

(10) a. What a copy cat you are! You phoned every alum I did. You solicited every alum I did. And you got a check from every alum I did!

b. No, I only PHONED every alum you did. The rest is nonsense.

The Kratzer (1991) approach to focus yields an analysis of (10) and other boundfocus ACD cases (the interested reader is referred to Kratzer's paper and invited to work it through). Schwarz (2000)'s framework, on the other hand, has considerable difficulty with these and related constructions, as we'll see shortly.

\subsection{Sloppy Identity and Schwarz (2000)}

As Schwarz (2000) shows, the distinction between ellipsis-only and pro-form accounts of VPE entails an important asymmetry in empirical coverage.

\footnotetext{
${ }^{6}$ Curiously, a free-focus reading analogous to Sue is the only $x$ who thinks Sue is smart seems absent (or at least highly unsalient) in these cases, a fact we return to below.
} 
(11) a. When John has to cook, he doesn't want to [ø cook]

b. When he has to CLEAN, he doesn't [ $\varnothing$ want to clean] (either)

Example (11) presents a challenge for ellipsis-only accounts of VPE—namely that there doesn't seem to exist any suitable antecedent for the elided VP in (11b). ${ }^{7}$ Schwarz suggests that ellipsis-only accounts might explain this fact in terms of a modified identity condition on ellipsis licensing. In particular (and informally) the constraint will have to be adjusted to allow any sub-constituent of a VP to elide if it has occurred previously in the discourse. In this case the relevant reading of (11b) results from separate elisions of want to and clean.

This approach represents a weakening of the identity constraint on ellipsis, and as Schwarz points out, it overgenerates:

a. When John has to cook, he doesn't want to cook

b. When he has to CLEAN, he doesn't [ ${ }_{\varnothing}^{*}$ want to clean] (either)

We may think of the representations in (11) and (12) as LFs. Modulo ellipsis marking, the LFs given in (11a) and (12a) are identical. This identity is due to the explicit instantiation at LF of (11a)'s elided VP cook-a general property of ellipsis-only accounts, as noted previously. However, if the identity constraint on ellipsis looks at LFs, it's then a mystery why (11b) is acceptable but (12b) is not: any ellipsis mechanism capable of licensing (11b) given (11a) licenses (12b) given (12a).

Schwarz proposes a set of alternative LFs in lieu of those given in (11):

$$
\begin{aligned}
& \text { a. [VP } \\
& \text { b. [ }\left[\mathrm{VP}^{+} \text {CLEAN] } \lambda_{2} \text { [When John has to } t_{2} \text {, he doesn't [VP want to } \Delta_{2} \text { ] }\right] \\
& \text { [When John has to } t_{1} \text {, he doesn't [VPø want to } \Delta_{1} \text { ] }
\end{aligned}
$$

In (13a) and (13b) the VPs cook and CLEAN have undergone QR - "+" denotes a type-lifted constituent (c.f. Partee and Rooth (1983)). A lambda operator binds (a) their respective traces and (b) a co-indexed silent $\langle e, t\rangle$-type pro-form, given by $\Delta$. This analysis has a couple desirable properties: (a) it doesn't suggest identical LFs for (13a) and (12a), and (b) it accordingly yields a suitable antecedent for the elided VP in (13b) without weakening the identity constraint on ellipsis. ${ }^{8}$

Most interestingly, as Schwarz points out, the LFs shown in (13) are parallel to those in (5) - the LFs that licensed the so-called "sloppy" reading of the pronoun in the latter's elided VP. In both instances, antecedent and elided VPs both host a pro-form $(\Delta, h i s)$ co-indexed with some trace $t$. Both pro-form and trace are bound by a lambda abstract, merging these two argument slots and thereby ensuring they

\footnotetext{
${ }^{7}$ The reader may object that representation (11b) runs afoul of the FCE. Suffice to say both Kratzer (1991)'s and Schwarz (2000) provide for this. The salient question here is purely a matter of syntax - in particular, the nature of the antecedent for ellipsis.

${ }^{8}$ Although note that here Schwarz relies on an ellipsis mechanism sensitive to identity of lexical content (modulo indices) rather than identity of interpretation, a necessary step since the antecedent and elided VPs will have different interpretations across assignment functions. Moreover, though this mechanism doesn't "see" indices, it is aided in this respect by the FCE, which rules out, for instance, a reading of the ellipsis clause such that John doesn't want to shop.
} 
receive identical interpretations (including, importantly, at each of the members of their respective propositional focus sets. In other words, like (5b), (13b) represents an instance of sloppy identity facilitated by argument-doubling binding.

\subsubsection{Transitive Verb Ellipsis}

Schwarz (2000) points to constructions which, though marginal, seem to function like transitive verb (TV) analogs of (11):

(14) a. When John cooks something, he doesn't say what he does [ø cook]

b. When he BAKES something, he doesn't $\varnothing$ either

Though (14a) is, in Schwarz's words, "degraded in isolation," (14b) nevertheless seems to possess a sloppy reading such that John doesn't say what he bakes when he bakes something.

Since Schwarz, following what might be termed a standard set of assumptions, views TV ellipsis (TVE) as reducible to VPE—c.f. May (1985)—this represents something of an unhappy result. Here's why: given this assumption, for a sloppy reading of (14b) to be available, Schwarz requires, as above, a VP-type variable trace in the ellipsis site of (14a) such that the antecedent for (14b)'s ellipsis is something like say what he does $\Delta_{1}$. Note, however, that (again, given a standard set of assumptions), (14a) should contain an extraction trace due to the movement of what out of object position. As Schwarz notes, variables can't host traces, and so this derivation is unviable. Implicitly ruled out here is the possibility that what's elided in these cases is a TV rather than a full VP. Note, moreover, that these sorts of assumptions preclude a Schwarzian analysis of bound-focus ACD constructions (Section 3.2), since in what might be termed "standard" syntactic treatments, ACD reduces to VPE. We return to both classes of constructions below.

\subsection{Wrap-Up}

In this section, ATB ellipsis, bound-focus, and sloppy identity constructions have been shown to suggest verb binding in the same ways as constructions in Section 2 suggested pronominal binding. In each of these areas, binding is indicated for both VPE and TVE. Kratzer and Scwharz's accounts both lack empirical coverage here. Kratzer (1991), by virtue of being what I've termed an "ellipsis-only" framework, does not account for the sloppy readings we saw in Section 3.3. Schwarz (2000), on the other hand, has trouble bringing binding to bear in cases of TVE. The account developed below attempts to address both of these deficiencies.

\section{4. "Free" VE}

It's generally assumed in the literature — cf. Hardt (1993), Kratzer (1991), Schwarz (2000) - that when a focused VP (or, alternatively, a VP which dominates some 
focus-bearing sub-expression) serves as an antecedent for ellipsis, only bound-focus readings are available. In this section I argue that certain contexts bring out freefocus readings for these sorts of expressions.

a. You're an enormous copycat at recess. What's more, you're never content to just copy me; you always insist on copying and then one-upping me. Yesterday after I climbed on the monkey-bars, you climbed on them and then did an elaborate dismount. After I made a 3-pointer, you made one and then proceeded to dunk.

b. In general, I suppose you're right. But here's a counterexample: yesterday I only [ $\mathrm{vp}_{i}$ SOMERSAULTED] after you did $\varnothing_{i}$. I didn't somersault and do a cartwheel or anything fancy like that.

(16) Your father only told you to $\left[\mathrm{Tv}_{i}\right.$ READ] every book he did $\varnothing_{i}$ as a boy. He didn't demand that you then write book reports on them, too!

Only if your ticket asks you, verbatim, to [ $\mathrm{VP}_{i}$ SIGN it] need you [ ${ }_{i}$ do so].

(18) a. What do you mean I'm making you look bad? I thought since you started working on your section's papers it would be alright to turn in mine to the professor.

b. No, only if I [ $\mathrm{TV}_{i}$ SUBMITTED] my papers should you have $\varnothing_{i}$ yours.

Context sets up the ellipsis clause of (15b) to have a reading such that somersault is the only $P \in D_{\langle e, t\rangle}^{c}$ such that (15b)'s speaker $P_{\text {past }}$ after he somersaulted. ${ }^{9}$ Here the salient alternatives to somersault include properties like do a cartwheel, and so the ellipsis clause functions as a felicitous denial of its speaker's perpetual one-upmanship without denying (crucially) that its speaker is nevertheless something of a copycat (the latter corresponding to the bound-focus reading). Example (16) works similarly. The intended reading here is such that read is the only $R \in D_{\langle e,\langle e, t\rangle\rangle}^{c}$ such that the child's father told him to $R$ every book he had read. The salient alternatives to read are transitive relations such as write a book report on, and so (16) denies the father's sadism rather than the proposition that he wants his child to do things he did as a boy. I won't discuss examples (17) and (18) other than to note that they provide additional evidence for free-focus ellipsis readings.

Importantly, these reading are analogous to the previously-noted free-focus reading of only $S U E_{i}$ thinks she ${ }_{i}$ is smart:

a. Sue is the only $x \in D_{e}^{c}$ s.t. $x$ thinks Sue is smart.

b. somersault is the only $P \in D_{\langle e, t\rangle}^{c}$ s.t. I $P_{\text {past }}$ after you somersaulted.

c. read is the only $R \in D_{\langle e,\langle e, t\rangle\rangle}^{c}$ s.t. your father told you to $R$ every book he read.

In each of these cases, a focused constituent serves as some sort of antecedent. In contrast to bound-focus readings, here the anaphorically-interpreted constituents remain invariant across the focus alternatives associated with their respective sentences (recall that in bound-focus readings, the anaphors co-varied with their respective antecedent sites across focus alternatives).

\footnotetext{
${ }^{9}$ Informants confirm this reading's availability.
} 
As noted previously, the bound/free distinction may, given certain assumptions, be taken to predict the existence of bound- and free-focus readings in pronominal constructions. In particular (and following much of the literature) we assumed that semantically identifying a pronominal with some focused antecedent $A_{F}$ via "free" co-reference does not cause the anaphor to inherit the set of alternatives associated with $A_{F}$. If ellipsis sites, like pronominals, may be interpreted as free anaphors, this likewise predicts free-focus readings for ellipsis constructions.

Kratzer (1991)'s framework is explicitly designed to restrict VE constructions with focused verbal antecedents to bound-focus readings and so runs afoul of these data. Schwarz (2000), however, can account for these data if he assumes that a focused constituent may serve as an antecedent for the elision of a non-focused constituent-remember that in Schwarz's framework binding supplements rather than replaces identity-based mechanisms for/constraints on ellipsis:

\section{I only SOMERSAULTED after you did [ø somersault]}

Your father only told you to READ every book he did [ $\varnothing$ read] as a boy

\section{The Framework}

An empirically adequate account of VE should, inter alia, (a) generate bound-focus readings for both VPE and ACD constructions (Section 3.2), (b) demonstrate an interpretive flexibility amenable to free-focus VPE and ACD readings (Section 4), (c) generate sloppy readings for Schwarz (2000)'s VPE constructions (Section 3.3), and (d) preferably have something to say about (1) the availability of a sloppy reading in Schwarz (2000)'s “degraded” TVE construction (Section 3.3.1), and (2) the relative acceptability and semantics of ATB ellipsis constructions (Section 3.1). ${ }^{10}$

Following Schwarz (2000), Jacobson (1992a), Szabolcsi (1992), and Hardt (1993), I propose a pro-form account of VE in the Combinatory Categorial Grammar (CCG) tradition—cf. Steedman (2000), Jacobson (1999), Barker (2005), and many others. Like Hardt (1993) and Jacobson (1992a), this account assumes that ellipsis resolution is in general a matter of semantically identifying a pro-verb with some suitable antecedent—one which, moveover, need not be overt. ${ }^{11}$ My account differs from these two in the following respect: semantic resolution of ellipsis sites can happen in two distinct ways - freely or via binding. This ambiguity reflects a melding of CCG frameworks for VE pioneered by Szabolcsi (1992)—who showed how to bind pro-VPs — and Jacobson (1992a) — who showed how to treat VPE and $\mathrm{ACD}$ as free anaphora in a variable-free logic.

\footnotetext{
${ }^{10}$ For those keeping score, Schwarz (2000) runs afoul of (a), (d1), and presumably (d2) (Jacobson (1996) shows that what might be termed "standard" accounts struggle to account for ATB pronominal binding without over-generating, a point I suspect will carry over to this domain). Kratzer (1991), by contrast, has trouble with (b), (c), (d1), and (again, presumably) (d2).

${ }^{11}$ Semantic accounts of ellipsis must, of course, end up being constrained somehow in order to avoid rank over-generation. Such a project lies beyond the goals of the present paper. I return to this worry briefly below.
} 
As with pronominals, this ambiguity predicts both free- and bound- focus readings as potentially viable interpretations of ellipsis clauses with focused VPand TV-type antecedents. Moreover, the syntactic assumptions of CCG and the mechanisms I employ for binding, when taken together, are such that analyses of ATB ellipsis, bound-focus TVE readings, and Schwarz's "degraded" TVE cases are direct consequences of the framework. Finally, since the account does not lead us to posit identical LFs for sentences like when John had to cook, he didn't want to and when John had to cook, he didn't want to cook, Schwarz's sloppy VPE cases do not present a difficulty.

\subsection{CCG and the Logic of Jacobson (1999)}

In CCG, a lexical item's distributional properties are encoded in its syntactic category, the argument structure of which maps directly onto that of its functional denotation. When some functor category receives its argument directly in the syntaxi.e. immediately to its right or left—the two strings concatenate. The denotation of the resulting constituent is arrived at via the semantics of function application (FA):

$$
\frac{\frac{X}{\mathrm{~A} / \mathrm{B}: f} \frac{Y}{\mathrm{~B}: b}}{\frac{X Y Y}{\mathrm{~A}: f b}} \text { FA }
$$

A note on notation: "A/B" denotes a functor which takes a constituent of category B to its right to yield a constituent of category A, whereas an $A \backslash B$ looks for something of category B to its left to yield a constituent of category A. Items with category $\mathrm{A}^{\mathrm{B}}$ distribute like items of category A but "host" unbound pro-forms of category B. Pronouns, for instance, are of category $\mathrm{NP}^{\mathrm{NP}}$ and function as identity maps:

$$
\frac{\operatorname{him}}{\mathrm{NP}^{\mathrm{NP}}: \lambda x[x]} \text { lex }
$$

Jacobson's CCG makes crucial use of type-shifting operations. Geach (g) a unary version of Curry and Feys's B-allows functors to inherit missing/gapped meanings from their arguments. Geaching shifts functors over items of category A into functors over items of category $A^{B}, A / B$, or $A \backslash B$. This has the effect of "passing up" the missingness of B while preserving the directionality in which B is sought. Since $\mathbf{g} a b c=a(b c), \mathbf{g}$ is a unary, Curry'd version of function composition ( $\alpha$ denotes a variable over syntactic expressions in the object language):

$$
\begin{array}{ccc}
\alpha_{\mathrm{C} / \mathrm{A}} & \Longrightarrow \mathbf{g} & \alpha_{\mathrm{C}^{\mathrm{B}} / \mathrm{A}^{\mathrm{B}}} \\
\alpha_{\mathrm{C} / \mathrm{A}} & \Longrightarrow \mathbf{g} & \alpha_{(\mathrm{C} / \mathrm{B}) /(\mathrm{A} / \mathrm{B})} \\
\lambda a[f(a)] & \lambda f \lambda g \lambda b[f(g b)] & \lambda g \lambda b[f(g b)]
\end{array}
$$

Like other CCG frameworks, Jacobson (1999) uses a lift operator T which reverses the function-argument relationship between a functor $f$ and argument $a$ : 


\begin{tabular}{ccc}
$\alpha_{\mathrm{A}}$ & $\Longrightarrow \mathbf{T}$ & $\alpha_{\mathrm{B} /(\mathrm{B} \backslash \mathrm{A})}$ \\
$a$ & $\lambda x \lambda f[f(x)]$ & $\lambda f[f(a)]$ \\
\hline$\alpha_{\mathrm{A}}$ & $\Longrightarrow \mathbf{T}$ & $\alpha_{\mathrm{B} \backslash(\mathrm{B} / \mathrm{A})}$ \\
$a$ & $\lambda x \lambda f[f(x)]$ & $\lambda f[f(a)]$
\end{tabular}

Together, $\mathbf{T}$ and $\mathbf{g}$ allow pronominal meanings to be passed up indefinitely. An example derivation follows for a case of free pronominal anaphora:

(22) $\operatorname{John}_{i} \operatorname{likes~him}_{j}$

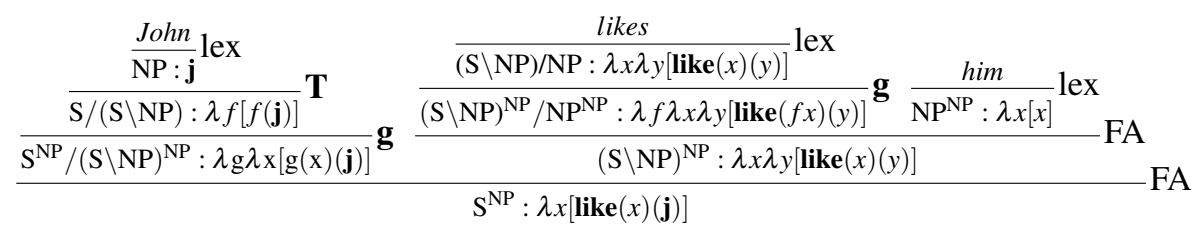

Likes looks for an NP to its right to form a VP. Finding an NPNP — an NP which contains an unbound pronominal — instead, it undergoes $\mathbf{g}$ to resolve a type and category mismatch (in the spirit of Partee and Rooth (1983)). Concatenating yields a constituent with the following category: $(\mathrm{S} \backslash \mathrm{NP})^{\mathrm{NP}}-\mathrm{a} \mathrm{VP}^{\mathrm{NP}}$. John lifts over VPs, undergoes $\mathrm{g}$-in effect composing with the $\mathrm{VP}^{\mathrm{NP}}$ - and an $\mathrm{S}^{\mathrm{NP}}$ results, denoting the property of being liked by John. The processor applies this meaning to a contextually salient individual to yield a proposition.

To bind pronouns, Jacobson (1999) deploys an argument-doubling typeshifter $\mathbf{z}$. The $\mathbf{z}$ rule applies directly to verbs rather than full VPs and, as such, is a more local, variable-free analog of the Derived VP rule:

$$
\begin{array}{ccc}
\alpha_{(\mathrm{A} \backslash \mathrm{B}) / \mathrm{C}} & \Longrightarrow_{\mathbf{z}} & \alpha_{(\mathrm{A} \backslash \mathrm{B}) / \mathrm{C}^{\mathrm{B}}} \\
\lambda c \lambda b[f(c)(b)] & \lambda f \lambda g \lambda b[f(g b)(b)] & \lambda g \lambda b[f(g b)(b)]
\end{array}
$$

Like $\mathbf{g}, \mathbf{z}$ allows a constituent $\alpha$ to combine with a $\mathrm{C}^{\mathrm{B}}$. Unlike $\mathbf{g}, \mathbf{z}$ binds the pronominal to $\mathbf{z} \alpha$ 's second argument. An example derivation follows for a case of quantificational pronominal binding:

(23) Every $\operatorname{man}_{i}$ likes his $_{i}$ mother.

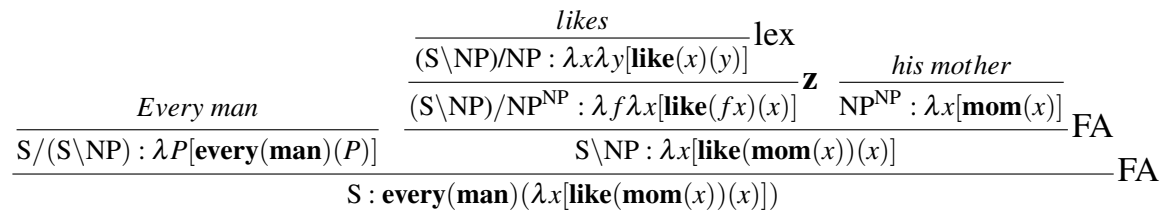

\subsection{VPE and ACD as Free Anaphora}

Jacobson (1992a) shows that with the addition of one assumption-in particular that auxiliaries, normally of category VP/VP, can shift into pro-form-hosting constituents of category VPVP — the Jacobson (1999) framework yields free-anaphora 
analyses of a wide range of VPE and ACD cases. ${ }^{12}$ In the case of VPE, the VPsuperscript is merely repeatedly passed up with $\mathbf{g}$ and $\mathbf{T}$. The resulting constituent is of category $\mathrm{S}^{\mathrm{VP}}$ and denotes a function from VP-type meanings to propositions:

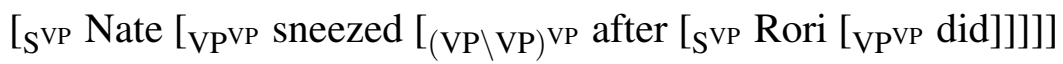

In these respects the Jacobson (1992a) derivation of VPE is parallel to the Jacobson (1999) derivation of "accidental" co-reference readings (as given for example 22).

Deriving free anaphoric ACD requires an additional step, but one which falls out of the framework. In particular, we apply $\mathbf{g}$ to pro-form does, as follows:

$$
\frac{\frac{\text { does }_{\text {pro }}}{\mathrm{VP}^{\mathrm{VP}}: \lambda P[\operatorname{does}(P)]}}{(\mathrm{VP} / \mathrm{NP})^{\mathrm{VP} / \mathrm{NP}}: \lambda R \lambda x[\operatorname{does}(R x)]} \mathbf{g}
$$

Since VP/NP is equivalent to category $\mathrm{TV},{ }^{13}$ the resulting category may be rewritten as $\mathrm{TV}^{\mathrm{TV}}$. So on this derivation does hosts an unbound pro-TV.

Let's see how this works for a fairly typical case of ACD:

Nate reads every book that Rori does $\varnothing$.

"Standard" accounts of ACD assume a trace in the ellipsis site corresponding to the extraction site for that. What elides, then, is necessarily something like read $t_{1}$. Moreover, since on these sorts of accounts quantified NPs cannot (generally speaking) be interpreted in object position, they undergo $\mathrm{QR}$, an operation which in the case of ACD yields an appropriate antecedent for ellipsis—c.f. May (1985):

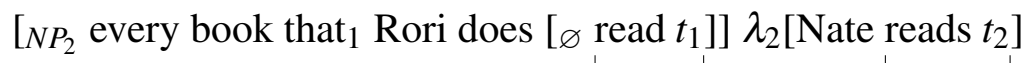

CCG, by contrast, doesn't require extraction gaps in order to compose relative clauses - cf. Steedman (1987), Jacobson (1992b), etc. Accordingly, the "missing" component in (25) is the transitive relation read-not read $t_{1}$. Jacobson (1992a) points out that since (a) a TV is what CCG requires at the ellipsis site for the syntax and semantics of relative clauses, and (b) the pro-verb category of does lets it "act" like a TV and pass up the pro-TV gap, we have a free anaphora analysis of ACD:

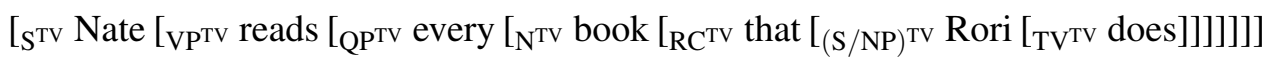

\subsection{VPE as Binding}

Szabolcsi (1992) shows how argument-doubling combinators like Curry and Fey's $\mathbf{W}$ or Jacobson's $\mathbf{z}$ yield binding analyses of elliptical phenomena. Either combinator can be used to derive the full range of Szabolcsi's data (and, indeed, Kratzer (1991)'s as well). I adopt $\mathbf{z}$ in the analysis here for expository reasons.

Recall one of Kratzer's bound-focus cases from before:

\footnotetext{
${ }^{12}$ This move seems to hold a fair amount of intuitive plausibility since elliptically-interpreted auxiliaries share many distributional properties with full VPs.

${ }^{13}$ In particular, both abbreviate category (S $\left.\backslash \mathrm{NP}\right) / \mathrm{NP}$.
} 
A plausible category for after might be (VP $\backslash \mathrm{VP}) / \mathrm{S}$ - that is, it takes a sentence to its right to yield a VP modifier. Shifting after with $\mathbf{z}$ yields a constituent of category $(\mathrm{VP} \backslash \mathrm{VP}) / \mathrm{S}^{\mathrm{VP}}$. So $\mathbf{z}($ after $)$ wants to take a sentence hosting a pro-VP and bind this pro-VP to its subsequent VP argument. Here, drove the pink Edsel binds both of these argument slots to yield a VP-type meaning equivalent to that of drove the pink Edsel after Jim drove the pink Edsel:

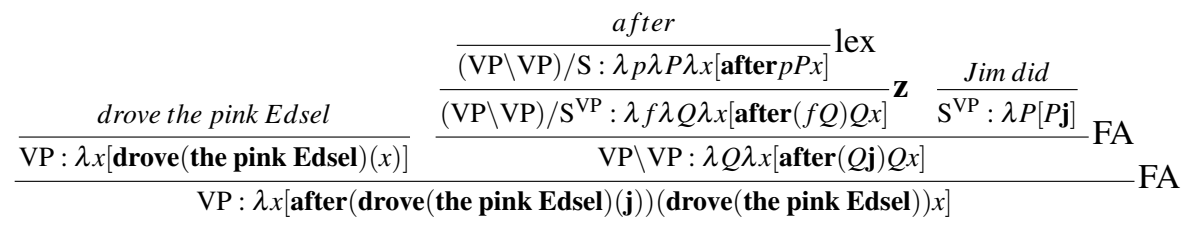

After $\mathbf{z}$ applies in the derivation, argument slots corresponding to the antecedent and elided VPs are merged, ensuring an unmixed focus set (such that antecedent and elided VPs co-vary on every focus alternative). A generalized version of the Jacobson (1999) framework - generalized in that $\mathbf{z}$ applies freely rather than to verbs exclusively—thus derives bound-focus readings without stipulation.

\subsection{A New Piece: ACD as Binding}

A binding analysis of ACD is likewise a consequence of generalizing Jacobson (1999)'s approach to anaphora. Here we require an additional piece of machinery: a special category for quantified objects. ${ }^{14}$ Quantified subjects are generally assumed to be of category S/VP. We stipulate here that quantified objects are ambiguous between category S/VP and VP $\backslash \mathrm{TV}$.

$$
\frac{\text { every book }}{\mathrm{VP} \backslash \mathrm{TV}: \lambda R \lambda y[\operatorname{every}(\mathbf{b o o k})(\lambda x[R x y])]} \text { obj }
$$

If here the quantified phrase every man has the category $\mathrm{VP} \backslash \mathrm{TV}$, it follows that the quantified determiner every be of category (VP $\backslash \mathrm{TV}$ )/ $\mathrm{N}$-in prose, something which takes a noun to its right to produce a left-looking quantified object.

This is all that's required for a binding analysis of ACD. We shift the quantified determiner by $\mathbf{z}$, yielding an item of category $(\mathrm{VP} \backslash \mathrm{TV}) / \mathrm{N}^{\mathrm{TV}}$. This constituent's denotation merges an unbound pro-TV in its nominal argument (corresponding to the deletion) with the main-clause TV:

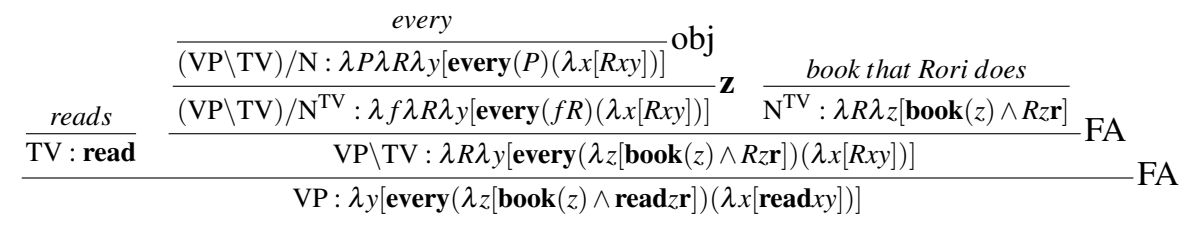

\footnotetext{
${ }^{14}$ Most if not all semantic theories require some extra apparatus in order to (a) accommodate quantified phrases in object position while (b) ensuring scopal ambiguity for expressions with both quantified subjects and objects. QR is one such example.
} 
The resulting function characterizes the set of individuals $y$ such that the set of books that Rori read is a subset of the set of things that $y$ read, which seems to be a reasonable denotation for the VP. As before, merging the pro-TV and overt TV argument slots guarantees an unmixed focus set, affording us an analysis of bound-focus ACD cases. ${ }^{15}$

\subsection{Deriving Schwarz (2000)'s Sloppy Readings}

Recall examples (11) and (12) from above, repeated here as (29) and (30):

(29) a. When John has to cook, he doesn't want to [ø cook]

b. When he has to CLEAN, he doesn't [ø want to clean] (either)

a. When John has to cook, he doesn't want to cook

b. When he has to CLEAN, he doesn't [ ${ }_{\varnothing}^{*}$ want to clean] (either)

The question these constructions posed for ellipsis-only accounts of VPE was, in short, why (29b) seemed to possess a sloppy reading that (30b) lacked. This was puzzling in light of the fact that within such frameworks (29a) and (29b) give rise to identical LFs. Since (29b) and (30b) are ostensibly identical, their respective (a) sentences should license the same range of possible interpretations for both.

Schwarz (2000) showed that treating some elided VPs as bound variables obviated these concerns. Specifically, he proposed that want to clean in (29b) be resolved in a complex fashion, with want to supplied via "normal" ellipsis mechanisms and clean via binding. If we think of "normal" ellipsis as corresponding to free anaphora and binding as corresponding to, well, binding, it turns out that the CCG framework we've adopted here has a similar story to tell.

First, we require a new piece for configurations like (29a) and (29b). In these constructions, the item we'll want to shift to facilitate binding is when John has to. However, if we conceive of when as a propositional operator-i.e. with category $(\mathrm{S} / \mathrm{S}) / \mathrm{S}^{16}$ - the category of when John has to is $(\mathrm{S} / \mathrm{S}) / \mathrm{VP}$, and its functional denotation is $\lambda P \lambda q[$ when $($ has-to $(P)(\mathbf{j}))(q)]$. Accordingly, we're looking to bind into this function's second argument (i.e. $q$ ) rather than its first. The $\mathbf{z}$ combinator, by contrast, is built for binding into some functor's first argument slot. ${ }^{17}$

In fact, $\mathbf{z}$ has a mirror image in Curry and Feys (1958)'s $\mathbf{S}$ (hence the name " $\mathbf{z}$ "). Various versions of $\mathbf{S}$ receive independent motivation in the CCG literaturee.g. Barker (2005), Russell (2005), Steedman (1987), and Szabolcsi (1992). The

\footnotetext{
${ }^{15}$ This derivation yields surface scope. Though inverse scope derivations of ACD binding exist in the framework under discussion, we won't have space to explore them here. Moreover, though the framework can be adapted to NP-S analyses of relative clauses in the spirit of Bach and Cooper (1978) and Cunningham (2007), I leave this discussion for another day.

${ }^{16}$ Here, when might instead be conceived of as a raised VP-modifier, eliminating the worry. However, since the issue returns with uncontroversial propositional operators such as if, the solution is worth pursuing.

${ }^{17}$ Importantly, I'm referring to the order of the arguments inside the scope of the $\lambda$ operators rather than the order in which the function itself takes these arguments.
} 
particular $\mathbf{S}$ rule given here- $\mathbf{S}^{\prime}$ - is a unary version of the forward substitution rule explored in Steedman (1987). It is structured so as to make the "binder" argument more "accessible" in the course of a derivation, in particular by making it $\mathbf{S}^{\prime} f$ 's first argument rather than its second. ${ }^{18}$ So $\mathbf{S} x y z=x z(y z)$, whereas $\mathbf{S}^{\prime} x z \underline{z}=x z(y z)$ (the underlining is used contrastively). This move aids in the exposition by allowing the derivations to fit together with a minimum of liftings and geachings. ${ }^{19}$

$$
\begin{array}{ccc}
\alpha_{(\mathrm{A} / \mathrm{C}) / \mathrm{B}} & \Longrightarrow \mathbf{S}^{\prime} & \alpha_{\left(\mathrm{A} / \mathrm{C}^{\mathrm{B}}\right) / \mathrm{B}} \\
\lambda b \lambda c[f(b)(c)] & \lambda f \lambda b \lambda g[f(b)(g b)] & \lambda b \lambda g[f(b)(g b)]
\end{array}
$$

To derive (29b) we begin by applying $\mathbf{S}^{\prime}$ to when John has to (as above) and combine the result with the VP clean:

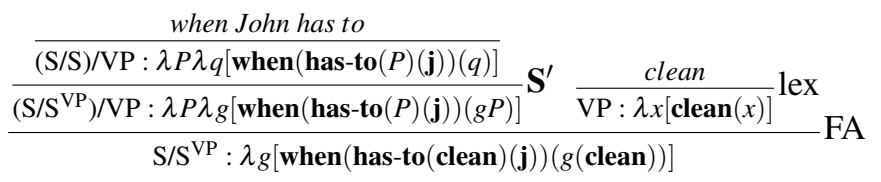

To derive he doesn't on the understanding such that what's elided isn't actually a full VP but rather a VPVP - i.e. want-we geach doesn't and combine the result with the NP John (ignoring the unbound pronominal), which requires lifting John and geaching the result twice:

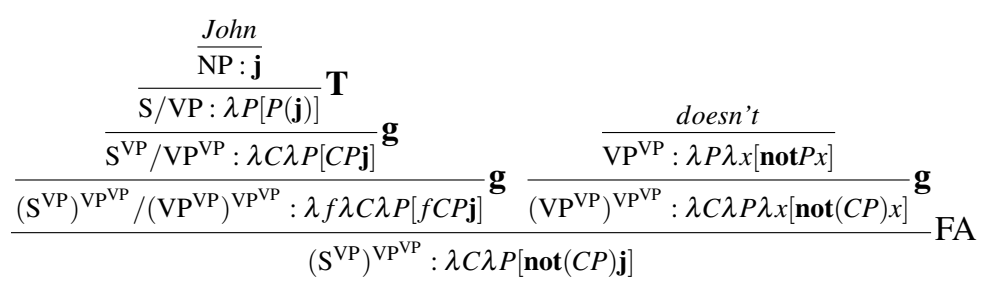

These two constituents subsequently compose, thereby passing up the free pro$\mathrm{VP}^{\mathrm{VP}}$ in he doesn't and binding off its free $\mathrm{VP}$ superscript (i.e. that of the $\mathrm{S}^{\mathrm{VP}}$ ):

$$
\frac{\frac{\text { when John has to clean }}{\mathrm{S} / \mathrm{S}^{\mathrm{VP}}: \lambda g[\text { when }(\text { has-to }(\text { clean })(\mathbf{j}))(g(\text { clean }))]} \frac{\text { John doesn't }}{\left(\mathrm{S}^{\mathrm{VP}}\right)^{\mathrm{VP}}: \lambda C \lambda P[\operatorname{not}(C P) \mathbf{j}]}}{\mathrm{S}^{\mathrm{VP}}: \lambda C[\text { when }(\text { has-to }(\text { clean })(\mathbf{j}))(\operatorname{not}(C(\text { clean })) \mathbf{j})]} \mathbf{B}
$$

In prose, this derivation binds off the pro-VP in the "consequent" clause but allows the unbound (i.e. superscripted) pro- $\mathrm{VP}^{\mathrm{VP}}$ to be passed up. So our resulting sentence is of category $\mathrm{S}^{\mathrm{VP}}$ and denotes a function from $\mathrm{VP}^{\mathrm{VP}}$-type meaningsspecifically, control-verb-like meanings-to propositions. ${ }^{20,21}$

\footnotetext{
${ }^{18}$ A similarly reordered version of $\mathbf{S}$ is adopted, for instance, in Barker (2005).

${ }^{19}$ Using $\mathbf{S}$ in lieu of $\mathbf{S}^{\prime}$ forces us to make use of the crossed composition rule $<\mathbf{B}_{\mathrm{x}}$, a non-orderpreserving combinator, but one which has been independently motivated for English in Steedman (1987) and Steedman (2000) among others.

${ }^{20}$ For (29b), the strict reading (such that when John has to clean, he doesn't want to cook) might be derived either by two instances of anaphoric resolution (i.e. the derivation yields a constituent of category $\left(\mathrm{S}^{\mathrm{VP}}\right)^{\mathrm{VP}}$ - to which the processor supplies want to and then cook) or one (i.e. the derivation yields an $\mathrm{S}^{\mathrm{VP}}$, and the relevant VP-type meaning is inferred in the vein of Webber (1978)).

${ }^{21}$ Note further that binding guarantees an unmixed focus set for (29b), satisfying the FCE.
} 
As to the lack of a sloppy reading for (30b), the analysis here shares a crucial property with that of Schwarz (2000): in constrast to (30a), at no level of representation does want in (29a) receive cook as an overt syntactic argument. Coupled with an empirically motivated generalization about what sorts of constituents represent viable antecedents for ellipsis, this derives the contrast between (29b) and (30b).

Since nothing prevents a derivation of $(30 \mathrm{~b})$ from proceeding in the same fashion as the above derivation of (29b) — and thus from likewise yielding an $\mathrm{S}^{\mathrm{VP}}$ the unavailability of the sloppy reading in the former case must be due to the unavailability of want as a possible elliptical meaning. Notice now that want doesn't make for a very good cross-sentential antecedent for ellipsis when it receives its rightward argument overtly in the syntax, a property it shares with TVs:

a. Bagels, I like. Donuts, I don't. ${ }^{22}$

b. I like bagels. ??I don't donuts.

a. To cook, I want. To clean, I don't.

b. I want to cook. ??I don't to clean.

The data is admittedly murky here. Notice, though, that the contrast between (29) and (30) follows if we accept the generalization (suggested by examples 31 and 32) that ceteris paribus rightward-looking functors (category $\beta / \gamma$ ) which receive their argument (category $\gamma$ ) adjacently in the syntax represent "bad" antecedents for ellipsis compared to rightward-looking functors which occur with an "overt gap"23 in lieu of $\beta$. This could conceivably be a fact about processing or the grammar, and at this point I see no a priori reason to come down in favor of one or the other. ${ }^{24}$

However, as this paper is not intended as a programmatic argument in favor of a semantics-only approach to free ellipsis resolution, if the reader remains unconvinced at this point (or alternatively finds this analysis descriptive rather than explanatory), I'm happy to concede a Schwarz-like syntactic identity-based mechanism for free ellipsis - embedded within the CCG account of syntax, semantics, and binding offered herein and in lieu of the "accidental" co-reference semantic ellipsis mechanism assumed previously. ${ }^{25}$ We still derive correct results for (29b) and (30b) by dint of not positing identical representations for (29a) and (30a) as input to the mechanism for ellipsis. ${ }^{26}$

${ }^{22}$ Construction due to Evans (1988).

${ }^{23}$ Terminology due to Chris Barker (pc).

${ }^{24}$ Like all semantic frameworks for ellipsis, ours needs to be fleshed out in one way or another (i.e. with appeals to processing, syntax, or both) to restrict the possible interpretations of ellipsis clauses more than it currently does. Conversely, syntactic constraints on ellipsis needs to be relaxed somewhat to (inter alia) accommodate gapping constructions and pragmatically controlled ellipsis.

${ }^{25}$ The relevant syntactic mechanism ensures string identity between antecedent and ellipsis material and is incapable of severing a rightward-looking functor of category $\beta / \gamma$ from an argument of category $\gamma$ if the two occur adjacently in the syntax (though we'll need to relax this so as not to rule out gapping and pseudo-gapping constructions). Semantic identity of antecedent and ellipsis material is still (as in Schwarz (2000)) guaranteed by the FCE.

${ }^{26}$ Indeed, the account of Schwarz (2000)'s sloppy cases offered here is in many ways the CCG relative of Schwarz's own. In both frameworks, the availability of both bound and free mechanisms for ellipsis is crucial for generating the sloppy reading of (29b). 


\subsection{ATB Ellipsis and Schwarz (2000)'s "Degraded" Constructions (Briefly)}

Here I present binding derivations of ATB ACD—example (7) above—and Schwarz's "degraded" TVE case—example (14). ${ }^{27}$

We begin with the ATB ACD construction John had to print ${ }_{i}$ and Mary had to file $j_{j}$ all the documents Sam neglected to $\varnothing_{i, j}$. Ignoring had to, we begin by deriving John printed, as follows: ${ }^{28}$

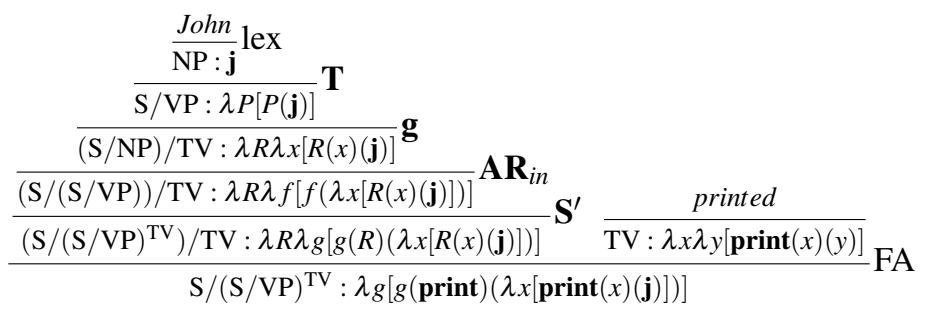

This yields something which takes a generalized quantifier hosting an unbound TV and returns a sentence. We derive Mary filed similarly and conjoin the two via the semantics of generalized conjunction — cf. Partee and Rooth (1983) — and apply the result to the (S/VP) ${ }^{\mathrm{TV}}$ all the documents Sam neglected to:

$$
\frac{\text { John printed and Mary filed }}{\frac{\mathrm{S} /(\mathrm{S} / \mathrm{VP})^{\mathrm{TV}}: \lambda g[g(\mathbf{p r i n t})(\lambda x[\operatorname{print}(x)(\mathbf{j})]) \wedge g(\mathbf{f i l e})(\lambda x[\mathbf{f i l e}(x)(\mathbf{m})])]}{\mathrm{S}: \operatorname{doc}(\boldsymbol{p r i n t})(\lambda x[\operatorname{print}(x)(\mathbf{j})]) \wedge \operatorname{doc}(\mathbf{f i l e})(\lambda x[\mathbf{f i l e}(x)(\mathbf{m})])} \frac{\text { all the documents Sam neglected to }}{(\mathrm{S} / \mathrm{VP})^{\mathrm{TV}}: \lambda R \lambda P[\operatorname{doc}(R)(P)]}} \text { FA }
$$

The derivation guarantees a meaning such that John had to print all the documents Sam neglected to print, and Mary had to file all the documents Sam neglected to file, a reasonable approximation of (6)'s truth conditions.

We turn now to deriving Schwarz's "degraded" TVE case. Doing so requires generalizing $\mathbf{S}^{\prime}$ as follows:

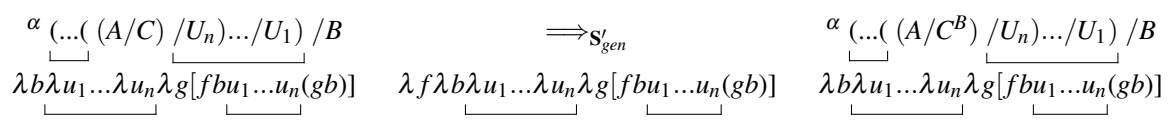

Underbrackets denote optional material. Generalized $\mathbf{S}^{\prime}$ therefore allows for an arbitrary number of intervening syntactic and semantic arguments between the binder and the thing being bound. Let's see how this works for when John BAKES something, he doesn't [ø say what he does bake] (example 14b). We begin by deriving when John bakes something: ${ }^{29}$

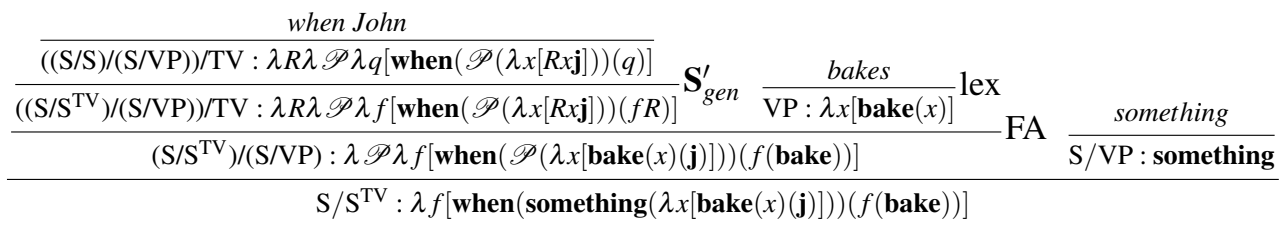

\footnotetext{
${ }^{27}$ The derivation of ATB VPE proceeds similarly and has been omitted due to space constraints.

${ }^{28}$ AR denotes Hendriks (1993)'s Argument Lift, an operation which allows a functor $f$ which wants some argument of type $\tau$ to shift into a functor $\mathbf{A R} f$, which may then combine with $\mathscr{T}$, an argument of type $\tau_{\text {lift }}$. AR receives substantial motivation in the CCG literature-e.g. Barker (2005). $\mathbf{A} \mathbf{R}_{i n}$, a geached version of $\mathbf{A R}$, allows us to apply this operation to inside arguments of $f$.

${ }^{29}$ The constituent when John has been derived here by lifting John and applying (respectively) $\mathbf{g}$, $\mathbf{A} \mathbf{R}_{\text {in }}$, and twice-geached when to the result.
} 
We derive he doesn't (ignoring the pronoun) with a pro- $\mathrm{VP}^{\mathrm{TV}}$ corresponding to say what he does and combine the two resulting expressions:

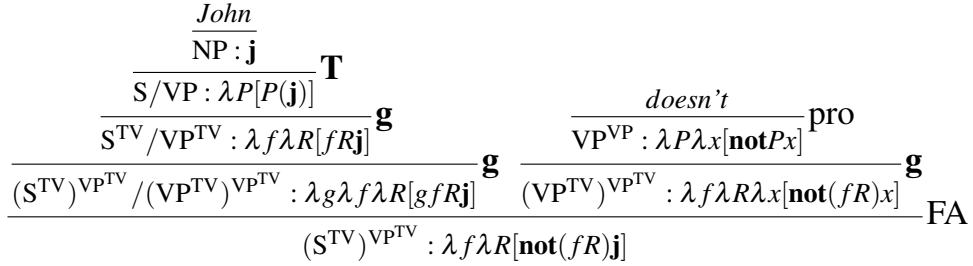

$$
\begin{aligned}
& \text { when John bakes something }
\end{aligned}
$$

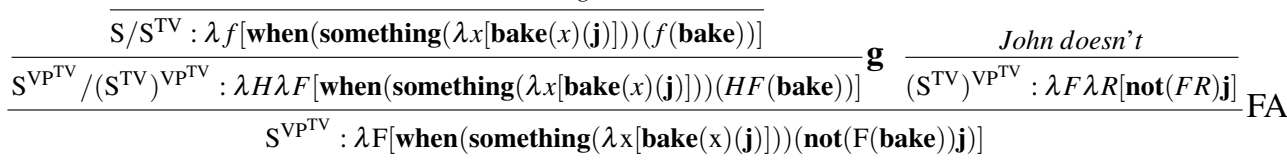

This yields an item of category $\mathrm{S}^{\mathrm{VP}}$ which maps objects of type $\langle\langle e, e t\rangle$,et $\rangle$ to propositions. Applying this function to say what he does yields a meaning such that when there exists some $x$ such that John bakes $x$, he doesn't say what he bakes. ${ }^{30}$ Binding again guarantees an unmixed focus set, thereby satisfying the FCE.

\section{Conclusion}

VPE and ACD share several properties with pronominal anaphora. All give rise to bound-focus readings, free-focus readings, sloppy identity under ellipsis, and ATB binding. I have provided examples and CCG analyses of VPE and ACD boundfocus, VPE and ACD free-focus, VPE and ACD sloppy identity, and ATB ACD (though not ATB VPE). The CCG analyses of these phenomena make use of the same apparatus the framework uses to analyze pronominal constructions.

\section{References}

Bach, Emmon and Robin Cooper: 1978, 'The NP-S Analysis of Relative Clauses and Compositional Semantics', Linguistics and Philosophy 2, 145-150.

Barker, Chris: 2005, 'Crossover as a Local Constraint', Linguistics and Philosophy 28, 447-472.

Cunningham, Emma: 2007, Relative Clauses, Domain Restrictions and Functional NPs, Unpublished ms., Brown University.

Curry, Haskell and Robert Feys: 1958, Combinatory Logic. North Holland Publishing Company, Amsterdam.

Evans, Frederic: 1988, 'Binding into Anaphoric Verb Phrases', in Proceedings of ESCOL (East Coast Conference on Linguistics). OSU Working Papers in Linguistics, Ohio State University.

\footnotetext{
${ }^{30} \mathrm{~A}$ derivation also exists with the quantified object taking scope over when. Though I believe this reading exists, space limitations preclude a proper treatment of this issue.
} 
Hardt, Daniel: 1993, VP Ellipsis: Form, Meaning, and Processing, Doctoral Dissertation, University of Pennsylvania.

Hendriks, Herman: 1993, Studied Flexibility. ILLC Dissertation Series, Amsterdam.

Jacobson, Pauline: 1992a, 'Antecedent contained deletion in a variable free semantics', in C. Barker and D. Dowty (eds.), Proceedings of SALT II. OSU Working Papers in Linguistics, Ohio State University.

Jacobson, Pauline: 1992b, 'The Lexical Entailment Theory of Control and the Tough Construction', in I. Sag and A. Szabolcsi (eds.), Lexical Matters. CSLI, Stanford.

Jacobson, Pauline: 1996, 'The Locality of Interpretation: The Case of Binding and Coordination', in T. Galloway and J. Spence (eds.), Proceedings of SALT VI. Cornell Working Papers in Linguistcs, Cornell.

Jacobson, Pauline: 1999, 'Towards a variable-free semantics', Linguistics and Philosophy 22, 117-184.

Kratzer, Angelika: 1991, 'The Representation of Focus', in A. von Stechow and D. Wunderlich (eds.), Semantics: An International Handbook of Contemporary Research, 825-832. de Gruyter, Berlin.

May, Robert: 1985, Logical Form: Its Structure and Derivation. MIT Press, Cambridge, MA.

Partee, Barbara and Mats Rooth: 1983, 'Generalized conjunction and type ambiguity', in R. Bäuerle, C. Schwarze, and A. von Stechow (eds.), Meaning, Use and Interpretation of Language. Walter de Gruyter, Berlin.

Partee, Barbara H.: 1975, 'Some transformational extensions of Montague grammar', in C. Hockney et al. (ed.), Contemporary Research in Philosophical Logic and Linguistic Semantics. Reidel, Dordrecht, Netherlands.

Rooth, Mats: 1985, Association with Focus, Doctoral Dissertation, UMass, Amherst.

Rooth, Mats: 1992, 'A Theory of Focus Interpretation', Natural Language Semantics 1, 75-116.

Russell, Benjamin: 2005, 'Functional Parasitic Gaps', in E. Georgala and J. Howell (eds.), Proceedings of SALT XV. CLC Publications, Ithaca, New York.

Sag, Ivan A.: 1976, Deletion and Logical Form, Doctoral Dissertation, Massachusetts Institute of Technology.

Schwarz, Bernhard: 2000, Topics in Ellipsis, Doctoral Dissertation, UMass, Amherst.

Steedman, Mark: 1987, 'Combinatory Grammars and Parasitic Gaps', Natural Language and Linguistic Theory 5, 403-439.

Steedman, Mark: 2000, The Syntactic Process. MIT press, Cambridge.

Szabolcsi, Anna: 1992, 'Combinatory Grammar and Projection from the Lexicon', in I. Sag and A. Szabolcsi (eds.), Lexical Matters. CSLI, Stanford.

Webber, Bonnie: 1978, A Formal Approach to Discourse Anaphora. Garland Press, New York.

Williams, Edwin: 1977, 'Discourse and logical form', Linguistic Inquiry 8, 101139. 